\title{
Paclitaxel Mortality in Peripheral Artery Disease
}

\author{
David Momtaz ${ }^{1}$, Abdullah Ghali ${ }^{1 *}$, Rishi Gonuguntla ${ }^{1}$, Hari Krishnakumar ${ }^{1}$, Greg Elder ${ }^{1}$, Boris Christopher ${ }^{1}$, Osama Altaee ${ }^{1}$ \\ ${ }^{1}$ University of Texas Health Science Center San Antonio
}

*Corresponding Author: Abdullah Ghali, University of Texas Health Science Center San Antonio.

Received date: April 10, 2021; Accepted date: April 18, 2021; Published date: April 28, 2021

Citation: David Momtaz, Abdullah Ghali, Rishi Gonuguntla, Hari Krishnakumar, Greg Elder, et.al. (2021). Paclitaxel Mortality in Peripheral Artery Disease. J Thoracic Disease and Cardiothoracic Surgery, 2(1); DOI:10.31579/2693-2156/019

Copyright: (c) 2021, Abdullah Ghali, This is an open access article distributed under the Creative Commons Attribution License, which permits unrestricted use, distribution, and reproduction in any medium, provided the original work is properly cited.

\begin{abstract}
Peripheral artery disease is a matter of global concern that affects 200 million people and is associated with decreased arterial perfusion in the extremities. The most plausible pathomechanism involves the formation of atheromas which subsequently cause occlusive atherosclerosis that impinge blood supply. Atheroma formation involves endothelial dysfunction with an accumulation of LDL (Low-density lipoprotein) that subsequently become oxidized and consumed by macrophages to form foam cells. The foam cells will release factors such as MMPs (Matrix metalloproteinases) and PDGF (platelet derived growth factor) that induce the proliferation and migration of smooth muscle cells, forming atheroma. Furthermore, endothelial cell damage can cause a loss of protective mechanisms, such as a reduction in the release of protective vasodilatory prostaglandins and Nitric Oxide. Atherosclerosis formation also decreases oxygen diffusion to the arterial media, resulting in atrophy in the vessel wall and ischemia. Additionally, chronic transmural inflammation cyclically releases increased MMPs and elastases that expand the arterial wall while degrading the protective collagen.
\end{abstract}

Keywords: atheromas; LDL; vasodilatory prostaglandins; nitric oxide; atherosclerosis; paclitaxel;

MMPs; elastases; peripheral artery; atheromas

\section{Introduction}

Peripheral artery disease is a matter of global concern that affects 200 million people and is associated with decreased arterial perfusion in the extremities [1]. The most plausible pathomechanism involves the formation of atheromas which subsequently cause occlusive atherosclerosis that impinge blood supply. Atheroma formation involves endothelial dysfunction with an accumulation of LDL (Low-density lipoprotein) that subsequently become oxidized and consumed by macrophages to form foam cells. The foam cells will release factors such as MMPs (Matrix metalloproteinases) and PDGF (platelet derived growth factor) that induce the proliferation and migration of smooth muscle cells, forming atheroma. Furthermore, endothelial cell damage can cause a loss of protective mechanisms, such as a reduction in the release of protective vasodilatory prostaglandins and Nitric Oxide. Atherosclerosis formation also decreases oxygen diffusion to the arterial media, resulting in atrophy in the vessel wall and ischemia. Additionally, chronic transmural inflammation cyclically releases increased MMPs and elastases that expand the arterial wall while degrading the protective collagen [2-3].

Paclitaxel is a ubiquitously used taxane, a plant diterpene, first isolated from the pacific yew and is commonly utilized as a chemotherapy drug. This plant-based microtubule inhibitor can stop the cell in the $\mathrm{M}$ phase, thereby halting cell division and proliferation. Paclitaxel is commonly used in the treatment of ovarian and breast carcinomas as well as a host of other neoplasms [4]. Paclitaxel works by hyperstablizing polymerized microtubules, inhibiting their breakdown and reassembly. The impact of these actions is a failure of anaphase to proceed, thereby halting the cycle of rapidly dividing cells. Some side effects can include myelosuppression, neuropathy, hypersensitivity, hair loss, muscle pains and diarrhea along with more serious adverse effects including heart problems, increased risk of infections and inflammation of the lung [5].

While it is well established that Paclitaxel can be used as a chemotherapeutic, at low concentrations there is reason to believe that at low concentrations it can have adverse effects. Low concentration of Paclitaxel can prevent restenosis, as well as limit the migration of smooth muscle cells, fibroblasts, and white blood cells out of the bone marrow [6]. Additionally, Paclitaxel is lipophilic, and is up taken very quickly and can remain present in the vessel walls at low concentrations for long periods [6]. In animal studies, Paclitaxel was present for 60 days after the initial exposure [7]. In cell culture, a 3 minute exposure to Paclitaxel led to decreased cell proliferation for 12 days [8]. Because of these qualities, one of the suspected risks of Paclitaxel use is that it can cause microenvironments of tumor spread [9], although additional research is required to elucidate the extent of the cellular compromise.

Paclitaxel is used as paclitaxel-covered balloons (PCD) and paclitaxeleluting stents (PES) when used in peripheral vascular interventions [9]. PCDs were first approved in 2012, and PESs were first approved in 2014 [9]. Randomly controlled trials have shown that usage of these devices improved short and long term procedural success when compared to devices that didn't have paclitaxel [9]. However, there isn't a great deal of research that has been done regarding the long-term safety of PCDs given the short amount of time they have been used.

Concerns regarding PCD's began to arise in 2018 when Katsanos et al. concluded there to be an increased risk of death following the application of placlitaxel-coated balloons in the femoropopliteal artery [10]. Though it was criticized for some flaws in its methodologies, the conclusions 
made by Katsanos were met with scrutiny and examination by the vascular community and scientists globally. This being epitomized by a statement released by the FDA in early 2019 citing meta-analysis studies identifying a late mortality signal in subjects treated with paclitaxelcoated devices. This statement went on to recommend caution when making treatment recommendations using PCD's and urged for continued data collection to further understand the long term safety and risk-benefit profile of PCD's [11]. In January 2021, the FDA released another statement citing a study from SWEDEPAD by Nordanstig et al., claiming there to be reassuring conclusions from this study which did not identify an increased mortality risk with PCD's [12].

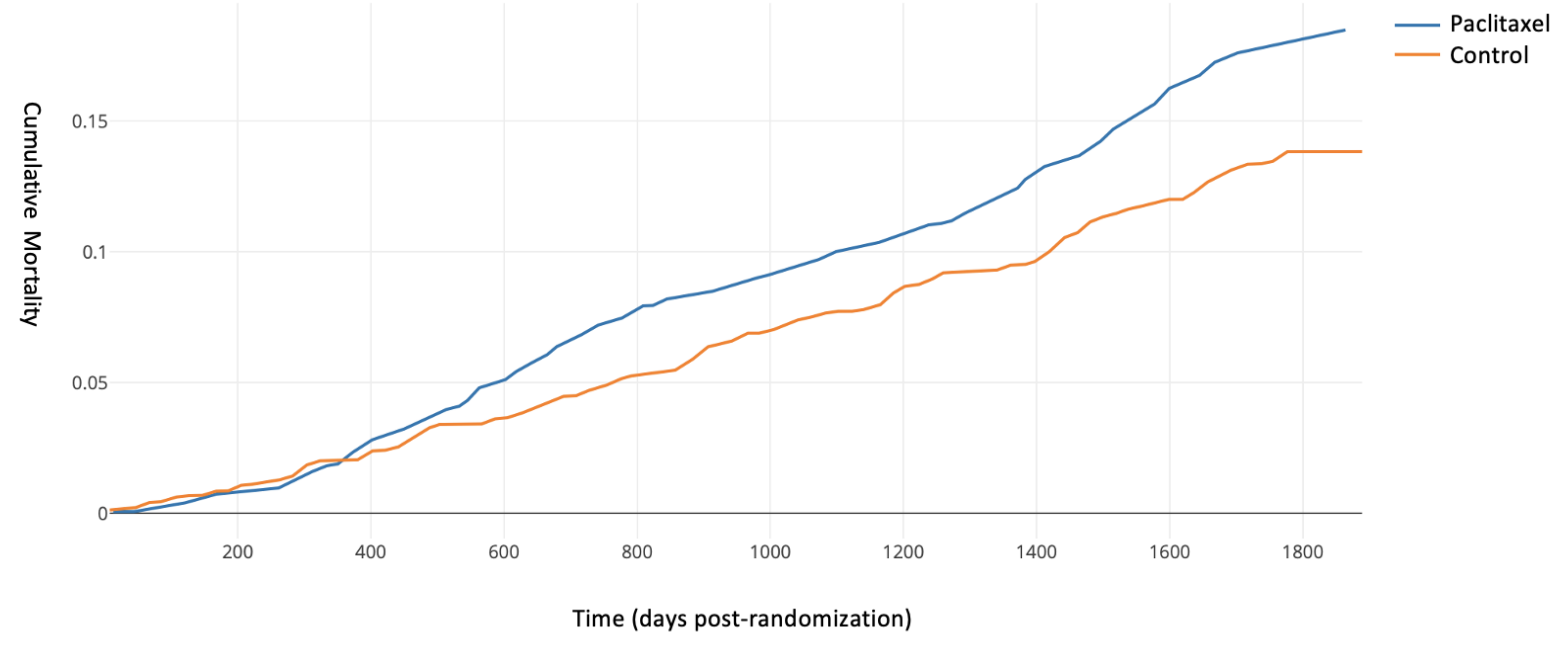

Figure 1. Cumulative mortality Vs Time for Paclitaxel and Control

Figure created from data obtained in the Rocha-Singh study. Singh RB, Mengi SA, Xu Y-J, Arneja AS, Dhalla NS. Pathogenesis of atherosclerosis: A multifactorial process. Experimental and clinical cardiology. 2002; 7(1): 40-53. Accessed December 26, 2020.

Nordanstig et al. is an unplanned randomized clinical trial with a total of 2289 randomly assigned patients. 1149 patients were assigned to the paclitaxel coated device group while 1140 were assigned to the control, uncoated, group. Patient follow up minimum was 1 year and maximum was 4 years, with a mean follow-up time of $\mathbf{2 . 4 9}$ years. During this time 574 total patients died, 281 from the uncoated group and 293 from the drug coated group. As a percentage, all-cause mortality among the uncoated device group was $9.9 \%$ while the drug coated group was calculated to be $10.2 \%$. The study concluded that there was no significant difference in the incidence of mortality between the treatment and control groups (of patients with chronic limb-threatening ischemia) or among those with intermittent claudication [13]. Figure 1 does indeed show that in one year post-randomization, the difference between the paclitaxel and control groups is minimal, and the difference between the two groups continues to be small for up to two years. However, the difference in cumulative mortality begins to pick up and steadily increase after the two year mark.

While Nordanstig et al. addresses a lack of a stated pathophysiology, in the Rocha-Singh study, a possible mechanism exists. Indeed, Paclitaxel's microtubule disrupting properties may directly impair protective mechanisms that are essential to endothelial cells proper function. Endothelial cilia, made of microtubule components, protect against atherosclerosis by promoting eNOS (endothelial nitric oxide synthase), an enzyme that is readily disrupted by atherosclerotic formation [14]. Furthermore, the Nordanstig et al. paper's no-mortality conclusion is congruent with other studies that follow short to midterm mortality [15]. The Swedish study describes patients being followed up for 1-4 years, with a mean length of 2.49 years. The length of follow-up could be a major determining factor in not observing increased mortality [15]. Figure
1 displays Paclitaxel all-cause mortality becoming more prominent with increased length of time, especially after the 2 year mark. It is also worth considering that Nordanstig et al.'s trial had a wide confidence interval that ranged from 0.72 to 1.93 . In comparison, the Rocha-Singh et al. paper boasts a $95 \%$ confidence interval displaying $6 \%-80 \%$ mortality. Thus, the attrition bias and or results-by-chance claims that Nordanstig et al. make in their paper are facile and not entirely supported with evidence.

Furthermore, Rocha-Singh provides a significantly more practical data set in the setting of American healthcare due to the fact that their metaanalysis only considers devices that have been US Food and Drug Administration-approved and commercially available in the United States. Meanwhile, Nordanstig considered all devices with European Union approval for peripheral artery disease interventions. Outside of there being a variable of different medical devices potentially affecting the results of these studies, it should also be noted that in the European Union, medical devices are approved by private bodies [16]. This has led to a trend of more high risk medical devices being approved faster, perhaps prematurely in the EU, which has led to a higher rate of device recalls in comparison to the US [16]. Disparities in the safety and regulation of medical devices further confounds conclusions from the Nordanstig study, while making the conclusions in Rocha-Singh more applicable and relevant to the American healthcare system.

Additional studies have been conducted that have concluded that Paclitaxel use is not correlated with higher rates of mortality. Schneider et al. is an independent patient level meta-analysis of 4 studies that concluded that there is no correlation between Paclitaxel use and mortality over an up to 5 year follow up period in a study of 1980 patients [17]. However, of these patients, only 143 did not receive PCDs, and of those patients, only 12 patients died during the monitoring time [17]. The small number of patients in this sample makes it difficult to draw strong conclusions. Rosenfeld et al. is a single-blind randomized control trial of 476 patients that found no correlation between Paclitaxel use and 
mortality [18]. However, this study only tracked 1 year outcomes, missing out on long term patient outcomes similarly to Katsanos et al. [18].

In conclusion, we believe that the shorter follow-up length, varied confidence intervals, and impact of an unstratified variable such as device safety are contributors to the observation of no difference in Paclitaxel mortality in the Nordanstig et al. study. We also believe that there exist plausible mechanistic processes by which Paclitaxel's adverse effects can be explained and that future research should focus on elucidating some of these possible mechanisms.

\section{References:}

1. Gul, F., \& Janzer, S. F. (2021). Peripheral Vascular Disease. In StatPearls. StatPearls Publishing.

2. Bergheanu, S. C., Bodde, M. C., \& Jukema, J. W. (2017). Pathophysiology and treatment of atherosclerosis: Current view and future perspective on lipoprotein modification treatment. Netherlands Heart Journal: Monthly Journal of the Netherlands Society of Cardiology and the Netherlands Heart Foundation, 25(4), 231-242.

3. Bergheanu, S. C., Bodde, M. C., \& Jukema, J. W. (2017). Pathophysiology and treatment of atherosclerosis: Current view and future perspective on lipoprotein modification treatment. Netherlands Heart Journal: Monthly Journal of the Netherlands Society of Cardiology and the Netherlands Heart Foundation, 25(4), 231-242.

4. Singh, R. B., Mengi, S. A., Xu, Y.-J., Arneja, A. S., \& Dhalla, N. S. (2002). Pathogenesis of atherosclerosis: A multifactorial process. Experimental \& Clinical Cardiology, 7(1), 40-53.

5. Bharadwaj, R., \& Yu, H. (2004). The spindle checkpoint, aneuploidy, and cancer. Oncogene, 23(11), 2016-2027.

6. Singla, A. K., Garg, A., \& Aggarwal, D. (2002). Paclitaxel and its formulations. International Journal of Pharmaceutics, 235(12), 179-192.

7. Gongora, C. A., Shibuya, M., Wessler, J. D., McGregor, J., Tellez, A., Cheng, Y., Conditt, G. B., Kaluza, G. L., \& Granada, J. F. (2015). Impact of Paclitaxel Dose on Tissue Pharmacokinetics and Vascular Healing: A Comparative DrugCoated Balloon Study in the Familial Hypercholesterolemic Swine Model of Superficial Femoral In-Stent Restenosis. JACC: Cardiovascular Interventions, 8(8), 1115-1123.

8. Krawisz, A. K., \& Secemsky, E. A. (2019). Paclitaxel-Based Devices for the Treatment of PAD: Balancing Clinical Efficacy with Possible Risk. Current Treatment Options in Cardiovascular Medicine, 21(10), 57.
9. Katsanos Konstantinos, Spiliopoulos Stavros, Kitrou Panagiotis, Krokidis Miltiadis, \& Karnabatidis Dimitrios. (2018). Risk of Death Following Application of Paclitaxel-Coated Balloons and Stents in the Femoropopliteal Artery of the Leg: A Systematic Review and Meta-Analysis of Randomized Controlled Trials. Journal of the American Heart Association, 7(24), e011245.

10. Health, C. for D. and R. (2019). August 7, 2019 UPDATE: Treatment of Peripheral Arterial Disease with Paclitaxel-Coated Balloons and Paclitaxel-Eluting Stents Potentially Associated with Increased Mortality. FDA.

11. Health, C. for D. and R. (2019). August 7, 2019 UPDATE: Treatment of Peripheral Arterial Disease with Paclitaxel-Coated Balloons and Paclitaxel-Eluting Stents Potentially Associated with Increased Mortality. FDA.

12. FDA Publishes Perspective on Paclitaxel-Coated Devices in PAD. Endovascular Today; Bryn Mawr Communications. Retrieved April 8, 2021.

13. Schermer, B., \& Benzing, T. (2016). Endothelial cilia protect against atherosclerosis. EMBO Reports, 17(2), 125-126.

14. Dinh, K., Gomes, M. L., Thomas, S. D., Paravastu, S. C. V., Holden, A., Schneider, P. A., \& Varcoe, R. L. (2020). Mortality after Paclitaxel-Coated Device Use in Patients with Chronic Limb-Threatening Ischemia: A Systematic Review and MetaAnalysis of Randomized Controlled Trials. Journal of Endovascular Therapy, 27(2), 175-185.

15. Rocha-Singh Krishna J., Duval Sue, Jaff Michael R., Schneider Peter A., Ansel Gary M., Lyden Sean P., Mullin Christopher M., Ioannidis John P.A., Misra Sanjay, Tzafriri Abraham R., Edelman Elazer R., Granada Juan F., White Christopher J., Beckman Joshua A., \& null null. (2020). Mortality and Paclitaxel-Coated Devices. Circulation, 141(23), 1859-1869.

16. Hwang, T. J., Sokolov, E., Franklin, J. M., \& Kesselheim, A. S. (2016). Comparison of rates of safety issues and reporting of trial outcomes for medical devices approved in the European Union and United States: Cohort study. The BMJ, 353.

17. Schneider Peter A., Laird John R., Doros Gheorghe, Gao Qi, Ansel Gary, Brodmann Marianne, Micari Antonio, Shishehbor Mehdi H., Tepe Gunnar, \& Zeller Thomas. (2019). Mortality Not Correlated With Paclitaxel Exposure. Journal of the American College of Cardiology, 73(20), 2550-2563.

18. Schneider Peter A., Laird John R., Doros Gheorghe, Gao Qi, Ansel Gary, Brodmann Marianne, Micari Antonio, Shishehbor Mehdi H., Tepe Gunnar, \& Zeller Thomas. (2019). Mortality Not Correlated With Paclitaxel Exposure. Journal of the American College of Cardiology, 73(20), 2550-2563. 